\title{
A longitudinal study of the association between dietary factors, serum lipids, and bone marrow lesions of the knee
}

\author{
Dawn Doré ${ }^{* \dagger}$, Jonathon de Hoog ${ }^{1 \dagger}$, Graham Giles ${ }^{2}$, Changhai Ding ${ }^{1,3}$, Flavia Cicuttini ${ }^{3}$ and Graeme Jones ${ }^{1}$
}

\begin{abstract}
Introduction: Bone marrow lesions (BMLs) play an important role in knee osteoarthritis, but their etiology is not well understood. The aim of this longitudinal study was to describe the association between dietary factors, serum lipids, and BMLs.
\end{abstract}

Methods: In total, 394 older men and women (mean age, 63 years; range, 52 to 79) were measured at baseline and approximately 2.7 years later. BMLs were determined by using $T_{2}$-weighted fat-saturation magnetic resonance imaging (MRI) by measuring the maximal area of the lesion. Nutrient intake (total energy, fat, carbohydrate, protein, and sugar) and serum lipids were assessed at baseline.

Results: Cross-sectionally, dietary factors and lipids were not significantly associated with BMLs. Energy, carbohydrate, and sugar intake (but not fat) were positively associated with a change in BML size $\beta=15.44$ to $19.27 \mathrm{~mm}^{2}$ per 1 SD increase; all $\left.P<0.05\right)$. High-density lipoprotein $(\mathrm{HDL})$ cholesterol tended to be negatively associated with BML change ( $\beta=-11.66 \mathrm{~mm}^{2}$ per $1 \mathrm{SD}$ increase; $P=0.088$ ).

Conclusions: Energy, carbohydrate, and sugar intake may be risk factors for BML development and progression. $\mathrm{HDL}$ cholesterol seems protective against BMLs. These results suggest that macronutrients and lipids may be important in BML etiology and that dietary modification may alter BML natural history.

\section{Introduction}

Osteoarthritis (OA) is a whole-organ disease characterized by gradual loss of articular cartilage. Strong evidence suggests that bone plays an important role in the pathogenesis of $\mathrm{OA}$, and it has been suggested that bone changes may precede cartilage damage [1]. Bone marrow lesions (BMLs), visible by using magnetic resonance imaging (MRI), have been recognized as a clinically important feature in OA [2,3]. A number of studies have linked BMLs with knee pain [2,4-6]. They are also associated with many structural changes in the knee, such as cartilage-defect progression $[7,8]$ and cartilage loss [7-10] on MR images, and they predict jointreplacement surgery $[6,11]$.

\footnotetext{
* Correspondence: Dawn.Dore@utas.edu.au

† Contributed equally

'Menzies Research Institute Tasmania, University of Tasmania, Private Bag 23, Hobart, Tasmania, 7000, Australia

Full list of author information is available at the end of the article
}

Growing evidence implicates nutritional factors in OA [12]. Specifically, nutrient and dietary supplements have been shown to be effective in relieving OA symptoms, and some may play a role in the course of the disease [13]. Elevated levels of fat and $n-6$ polyunsaturated fatty acids have been found in human OA bone [14]; whereas n-3 polyunsaturated fatty acids have been shown to modulate catabolic factors in articular cartilage destruction [15]. Recent studies have begun to examine the relation between fatty acids and BMLs. Wang et al. [16] reported that higher intakes of monounsaturated, total, and $n-6$ polyunsaturated fatty acids were associated with BMLs cross-sectionally [16]. In a recent longitudinal design, they showed that increased saturated fat intake was associated with incident BMLs [17]. These results require confirmation in different settings.

Although many attempts have been made to establish a relation between food and OA [13], to the best of our knowledge, no study has examined whether dietary components other than fat intake, such as total energy,
C Biomed Central

C 2012 Doré et al; licensee BioMed Central Ltd. This is an open access article distributed under the terms of the Creative Commons Attribution License (http://creativecommons.org/licenses/by/2.0), which permits unrestricted use, distribution, and reproduction in any medium, provided the original work is properly cited. 
protein, carbohydrate, and/or sugar intake are associated with BMLs.

Research has shown that the prevalence of vascular disease is high among people with OA [14,18]. Evidence suggests that these diseases may share risk factors, such as obesity, hypertension, high low-density lipoprotein (LDL) levels, elevated total cholesterol, diabetes, smoking, and diet [14,18-21]. Vascular pathology may contribute to the development of OA through its effects on the subchondral bone. Blood flow through the small vessels in the subchondral bone may be reduced by venous occlusion, which results in impaired venous circulation underlying the cartilage plate, joint hypertension, hypercoagulability, and/or microemboli [19]. These may result in subchondral bone ischemia, which can contribute to decreased nutrient supply to the overlying cartilage plate [19]. Subchondral bone ischemia can also affect osteocyte death, leading to bone resorption, reducing the viability of subchondral bone $[19,22]$. BML histology is heterogeneous and includes osteonecrosis, edema, trabecular abnormalities, and bone remodeling [23]. Additional MRI-histologic correlation studies of these lesions have demonstrated fat cell destruction and fibrovascular regeneration in the lesion area [24], as well bone marrow fibrosis in well-defined subchondral zones of OA [25]. Hunter et al. [26] demonstrated that BMLs are sclerotic compared with unaffected regions from the same individual, based on the increased bone-volume fraction and increased trabecular thickness. Recently, Leydet-Quilici et al. [27] showed that BMLs can be separated into edema-like and necrosis-like on MR images. Edema-like MR patterns were associated with histologic edema and, to a lesser extent, vascular fibrosis, whereas necrosis-like MR patterns were associated with histologic necrosis combined with fibrosis [27]. BMLs have also been linked to ischemia and/or reperfusion injury $[22,28]$. Therefore, it is possible that vascular pathology may influence BML development. To our knowledge, only one study examined serum lipids and BMLs, reporting that serum cholesterol and triglyceride levels were associated with an increased incidence of BMLs [29]. However, this study was conducted in asymptomatic women; therefore, further studies are needed in different populations to confirm this finding. Additionally, we do not know whether serum lipids are associated with BML progression.

The aim of this study, therefore, was to describe the association between dietary factors, serum lipids, and BMLs in a population-based sample of older adults.

\section{Materials and methods Subjects}

This study was conducted as part of the Tasmanian Older Adult Cohort (TASOAC) study, an ongoing prospective, population-based study aimed at identifying the environmental, genetic, and biochemical factors associated with the development and progression of OA at multiple sites (hand, knee, hip, and spine). Subjects between the ages of 50 and 80 years were randomly selected from the electoral roll in Southern Tasmania (population, 229,000), with an equal number of men and women. The overall response rate was $57 \%$. As TASOAC was designed to examine community-dwelling older adults, institutionalized older adults were excluded. Participants also were excluded if they reported contraindications for MRI. Of all initially eligible participants, 1,100 enrolled in the study, and 1,099 attended a baseline clinic between March 2002 and September 2004. Follow-up data were collected for 875 eligible participants at a subsequent clinic approximately 2 to 3 years later. The MRI machine was decommissioned halfway through the follow-up period; therefore, MRI scans were available for only approximately half of the follow-up participants $(n=425$ of 875). The current study consists of a sample of 394 TASOAC participants who had MRI measures at baseline and follow-up and dietary and lipid measures at baseline.

All research conducted was in compliance with the Declaration of Helsinki and was approved by the Southern Tasmanian Health and Medical Human Research Ethics Committee. All subjects gave informed written consent.

\section{Baseline anthropometrics and questionnaire}

Weight was measured to the nearest $0.1 \mathrm{~kg}$ (with shoes, socks, and bulky clothing removed) by using a single pair of electronic scales (Seca Delta Model 707). Height was measured to the nearest $0.1 \mathrm{~cm}$ (with shoes and socks removed) by using a stadiometer. Body mass index (BMI) was calculated as kilograms per square meter. Self-report of smoking status, statin use, and disease status, such as cardiovascular disease and diabetes, was recorded by questionnaire.

\section{Magnetic resonance imaging}

MRI of the right knee was acquired at baseline and follow-up with a 1.5 - $\mathrm{T}$ whole-body magnetic resonance unit (Picker, Cleveland, OH, USA) by using a commercial transmit/receive extremity coil. Image sequences included the following: (a) a $\mathrm{T}_{1}$-weighted fat-saturation three-dimensional (3D) gradient-recalled acquisition in the steady state; flip angle, 30 degrees; repetition time, 31 milliseconds; echo time, $6.71 \mathrm{~ms}$; field of view, 16 $\mathrm{cm}$; 60 partitions, $512 \times 512$-pixel matrix; acquisition time, 5 minutes 58 seconds; one acquisition; sagittal images were obtained at a slice thickness of $1.5 \mathrm{~mm}$ without a interslice gap; and (b) a $\mathrm{T}_{2}$-weighted fatsaturation two-dimensional (2D) fast spin echo, flip 
angle, 90 degrees; repetition time, 3,067 milliseconds; echo time, 112 milliseconds; field of view, $16 \mathrm{~cm}, 15$ partitions, $228 \times 256$-pixel matrix; sagittal images were obtained at a slice thickness of $4 \mathrm{~mm}$ with an interslice gap of 0.5 to $1.0 \mathrm{~mm}$.

\section{Bone marrow lesions}

Subchondral BMLs were assessed on $\mathrm{T}_{2}$-weighted MR images by using Osiris software and defined as areas of increased signal adjacent to the subcortical bone at the medial tibial, medial femoral, lateral tibial, and lateral femoral sites. One trained observer scored the BMLs by measuring the maximal area of the lesion at baseline and follow-up, as previously described [6]. The observer manually selected the MRI slice with the greatest BML size. The BML with the highest score was used if more than one lesion was present at the same site. The maximal area was measured in square millimeters by using software cursors. Baseline and follow-up MRIs were read paired with the chronologic order known to the observer. Intraobserver repeatability of our areal BML measurement was assessed by randomly selecting 40 subjects with a BML to have their MRI scans re-read after at least a 2-week interval. The reader was blinded to the original BML measurements. Each BML present was remeasured at the medial tibial, medial femoral, lateral tibial, and lateral femoral sites. We compared the areal measurement 1 with the areal measurement 2 at each site where a BML was present. The intraclass correlation coefficient (ICC) was 0.97 . At baseline and follow-up, participants were given a BML score (in square millimeters) at the medial tibial, medial femoral, lateral tibial, and lateral femoral sites. These were summed to create a total BML score. Figure 1(a and b) illustrates a change in BML size from baseline to follow-up.
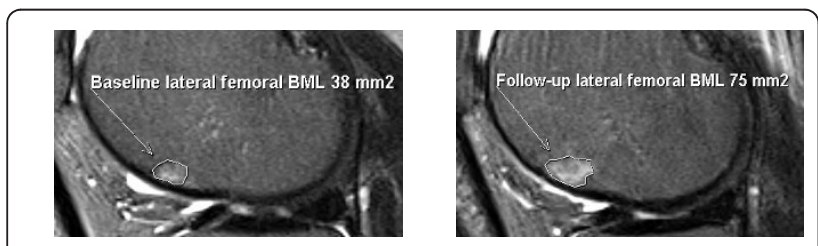

(A)
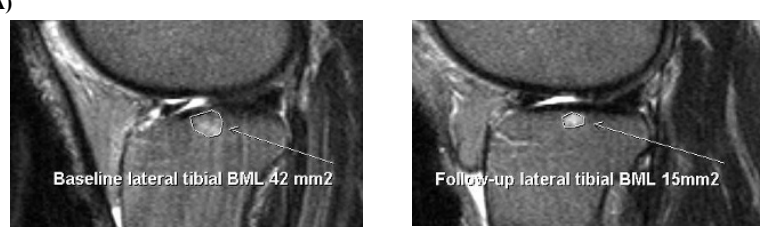

(B)

Figure 1 Examples of bone marrow lesion (BML) change. (a) BML increase from baseline to follow-up. (b) BML decrease from baseline to follow-up [6].

\section{Dietary factors}

Baseline dietary information was collected with the use of a self-administered, 74-item validated food-frequency questionnaire (FFQ) that was developed specifically for use in Australian adults [30,31]. Ten possible frequency responses were available for each food item, ranging from "never or less than once per month" to " 3 or more times per day." This information was used to compute specific nutrient intakes, such as total energy ( $\mathrm{kJ} /$ day), fatty acid (total fats, monounsaturated, polyunsaturated, and saturated (g/day)), carbohydrate (g/day), protein (g/ day), and sugar (g/day).

Information regarding over-the-counter medication included information about vitamin and mineral supplementation and natural and herbal medications. We have chosen not to include these as part of nutrient intake in the current study because supplements have become more complex, with different brands containing highly variable ingredient combinations. As a result, evidence now suggests that brief and simple questionnaires (those that have been used in the current study) do not accurately reflect supplement intake, and more validated methods are necessary [32]. Therefore, we limited our definition of nutrient intake to those data obtained from the FFQ.

\section{Serum lipids}

Blood samples were collected at baseline after a 12-hour overnight fast, and assays were conducted to measure enzymatically the total cholesterol, high-density lipoprotein (HDL) cholesterol, and triglycerides by using an Olympus AU5400 automated analyzer. The concentration of LDL cholesterol was calculated by using the Friedewald formula [33]. Assays were performed on thawed blood samples.

\section{Radiographic osteoarthritis}

A standing anteroposterior semiflexed view of the right knee with 15 degrees of fixed knee flexion was performed at baseline and scored individually for osteophytes and joint-space narrowing (JSN) on a scale of 0 to $3(0=$ normal, and $3=$ severe $)$ according to the Altman atlas [34], as previously described [35]. The presence of radiographic OA (ROA) was defined as any score $\geq 1$ for JSN or osteophytes.

\section{Additional available data}

Cartilage defects were assessed on $\mathrm{T}_{1}$-weighted $\mathrm{MR}$ images (score range, 0 to 4 ) at the medial tibial, medial femoral, lateral tibial, and lateral femoral sites, as previously described [36], as follows: grade $0=$ normal cartilage; grade $1=$ focal blistering and intracartilaginous low-signal-intensity area with an intact surface and base; grade 2 = irregularities on the surface or base and loss 
of thickness $<50 \%$; grade $3=$ deep ulceration with loss of thickness > 50\%; and grade $4=$ full-thickness chondral wear with exposure of subchondral bone. A cartilage defect also had to be present on at least two consecutive slices. The cartilage was considered to be normal if the band of intermediate signal intensity had a uniform thickness. The highest score was used if more than one defect was present on the same site.

Meniscal damage evaluation was performed by a trained observer, as previously described [37]. In brief, the proportion of the menisci affected by a tear or a partial or full extrusion was separately scored (yes/no) on the medial and lateral edges of the tibiofemoral joint at the anterior, middle, and posterior horns.

PA was assessed as steps per day, as determined by pedometer (Omron HJ-003 and HJ-102; Omron Healthcare, Kyoto, Japan), as previously described [35]. In brief, each participant was told to wear the pedometer for 7 consecutive days and to record the number of steps each day and the duration and type of PA for any activities in which the pedometer could not be worn (for example, swimming). An average of the 7 days was used to give each participant a mean steps-per-day value. Participants were then mailed a second pedometer after a 6-month period and repeated the process. A steps-per-day value, which was an average of the steps per day in summer and winter, was calculated.

\section{Data analysis}

The $t$ tests and $\chi^{2}$ tests were used to compare differences in means and proportions, as appropriate. Logistic regression was used to examine the associations between baseline BMLs (absent versus present) with baseline dietary factors and lipids after adjustment for age, sex, BMI, smoking, cardiovascular diseases, diabetes, ROA, and statin use in the lipids model.

Mixed-effect models were used to examine the association between change in total BML area and baseline dietary factors and lipids after adjustment for age, sex, BMI, baseline BMLs, smoking, cardiovascular diseases, diabetes, ROA, time to follow-up, and statin use in the lipids model. Standard diagnostic checks of model adequacy and unusual observations were performed and revealed that change in BML area was not normally distributed. The data are clumped at zero because a large proportion of participants did not have a BML at baseline or follow-up. However, the distribution remains not normal even after box-cox transformation. As a result, we have analyzed change in BML area as it was, but performed two separate analyses examining (a) BML size change at all four sites (medial tibial, medial femoral, lateral tibial, and lateral femoral); and (b) total BML size change (all four sites combined). This was done to check the consistency of our results.
Logistic regression analysis was used to examine the association between BMLs that have completely resolved and dietary factors and lipids after adjustment for age, sex, BMI, ROA, and statin use in the lipids model.

To compare with a previous study [17], a separate analysis examined only incident BMLs with baseline dietary factors and lipids. As we used a continuous, quantitative measure of BML size, we defined incident BMLs based on the least-significant criterion (LSC) [38]. We used this equation in a recent article [6] to define significant changes in BML size when assessing BMLs as a continuous measure. The LSC takes into account measurement error and the correlation between the BML measurements at baseline and follow-up. The formula was as follows:

$$
L S C=1.96 \times \sigma \sqrt{2(1-\rho)}
$$

where $\sigma$ is the standard error of the mean, and $\rho$ is the serial correlation. LSC was calculated to be $25 \mathrm{~mm}^{2}$ (where $\sigma=11.67$ and $\rho=0.38$ ). Therefore, incident BMLs were defined as any new BML in those with no BMLs at baseline larger than $25 \mathrm{~mm}^{2}$. Logistic regression analysis was performed to examine incident BMLs with dietary factors and lipids after adjustment for age, sex, BMI, ROA, and statin use in the lipids model.

Standard diagnostic checks of model adequacy and unusual observations were performed for all models. Hosmer-Lemeshow tests were performed to assess goodness-of-fit for the logistic regression models. The result for each logistic regression was $>0.05$, indicating that the model fits were adequate. Each dietary factor was entered into a separate multivariable model to avoid collinearity. A $P$ value less than 0.05 (two-tailed) was considered statistically significant. All statistical analyses were performed on Intercooled Stata 10.0 for Windows (StataCorp LP).

\section{Results}

\section{Subjects}

In total, 1,099 subjects (51\% female) aged between 51 and 81 years (mean, 63 years) participated in the TASOAC study. The current study consists of a sample of 394 TASOAC participants who had MRI measures at baseline and follow-up (approximately 2.7 years) and dietary and lipid measures at baseline. The range of follow-up times was 2.0 to 4.7 years. The majority of participants (90\%) were followed up between 2.2 and 3.2 years. No significant differences were found in demographics, baseline energy intake, fatty acid intake, carbohydrate intake, protein intake, sugar intake, total cholesterol, triglycerides, LDL cholesterol, HDL cholesterol, ROA, or statin use between the rest of the cohort $(n=705)$ and the subjects included in the current study 
$(n=394)$. The characteristics of the study population are presented in Table 1 . Those participants with a BML present at baseline $(n=0.168)$ were more likely to be male $(P=0.02)$ and had a higher BMI $(P=0.01)$ and a lower total and HDL cholesterol $(P=0.01)$. Total BML area at baseline ranged from 0 to $727 \mathrm{~mm}^{2}$. The change in total BML area ranged from -710 to 1,344 $\mathrm{mm}^{2}$.

Statin use was not associated with baseline BMLs (OR, 1.22; $P=0.452)$; however, those taking statin medication had a significantly lower total cholesterol level (OR, $0.51 ; P<0.001)$, higher triglyceride level (OR, 1.64; $P<$ 0.001 ), and lower LDL cholesterol level (OR, 0.23; $P<$ 0.001 ); therefore, we adjusted for statin use in the multivariable analysis examining lipids.

\section{Cross-sectional results}

Table 2 documents the associations between baseline BMLs with dietary factors and lipids. Dietary factors and

Table 1 Characteristics of participants according to presence or absence of BMLs at baseline

\begin{tabular}{|c|c|c|c|}
\hline & $\begin{array}{l}\text { BML } \\
\text { absent } \\
(n=226)\end{array}$ & $\begin{array}{l}\text { BML } \\
\text { present } \\
(n=168)\end{array}$ & $P$ \\
\hline Age (year) & $63.2(7.3)$ & $63.2(7.2)$ & 0.99 \\
\hline Female sex (\%) & 56 & 44 & 0.02 \\
\hline $\mathrm{BMI}\left(\mathrm{kg} / \mathrm{m}^{2}\right)$ & $27.1(4.0)$ & $28.3(5.0)$ & 0.01 \\
\hline Current smokers (\%) & 13 & 8 & 0.13 \\
\hline Cardiovascular disease (\%) & 4 & 9 & 0.07 \\
\hline Diabetes (\%) & 8 & 6 & 0.36 \\
\hline Statin use (\%) & 17 & 20 & 0.45 \\
\hline ROA present (\%) & 57 & 59 & 0.70 \\
\hline \multicolumn{4}{|l|}{ Dietary factors } \\
\hline Energy Intake (kJ/day) & $\begin{array}{l}7,512 \\
(2,378)\end{array}$ & $7,800(2,941)$ & 0.30 \\
\hline Total fat (g/day) & $71.5(25.9)$ & $74.2(34.5)$ & 0.40 \\
\hline $\begin{array}{l}\text { Monounsaturated fat (g/ } \\
\text { day) }\end{array}$ & $24.9(9.8)$ & $25.7(12.8)$ & 0.50 \\
\hline Polyunsaturated fat (g/day) & $11.8(5.0)$ & $11.6(6.1)$ & 0.67 \\
\hline Saturated fat (g/day) & $28.6(12.3)$ & $30.4(15.2)$ & 0.19 \\
\hline Total carbohydrates (g/day) & $205.1(67.8)$ & $214.3(76.1)$ & 0.21 \\
\hline Total protein (g/day) & $84.7(31.4)$ & $87.2(43.1)$ & 0.52 \\
\hline Total sugar (g/day) & $95.6(36.3)$ & $100.5(42.0)$ & 0.22 \\
\hline \multicolumn{4}{|l|}{ Lipids } \\
\hline Total cholesterol $(\mathrm{mmol} / \mathrm{L})$ & $5.7(1.1)$ & $5.4(1.1)$ & 0.01 \\
\hline Triglycerides (mmol/L) & $1.5(0.9)$ & $1.5(0.8)$ & 0.99 \\
\hline LDL cholesterol (mmol/L) & $3.6(0.9)$ & $3.5(1.0)$ & 0.10 \\
\hline HDL cholesterol (mmol/L) & $1.4(0.4)$ & $1.3(0.3)$ & 0.01 \\
\hline
\end{tabular}

Values expressed as mean (standard deviation), except for percentages. $P$ values determined by $t$ test or $\chi^{2}$ test (where appropriate). Boldface denotes a statistically significant result. BMI, body mass index; BMLs, bone marrow lesions; $\mathrm{HDL}$, high-density lipoprotein; LDL, low-density lipoprotein; ROA, radiographic osteoarthritis. lipids were not significantly associated with baseline BMLs in a multivariable model.

\section{BML change}

The association between baseline dietary factors and lipids with change in total BML size is presented in Table 3. Change in BMLs was significantly positively associated with energy, carbohydrate, and sugar intake in a multivariable model, adjusting for age, sex, BMI, baseline BMLs, smoking, cardiovascular diseases, diabetes, ROA, and time to follow-up. No significant associations were found between total fat intake or individual fats (monounsaturated, polyunsaturated, and saturated fat) and BML change. After adjustment for age, sex, BMI, baseline BMLs, smoking, cardiovascular diseases, diabetes, ROA, time to follow-up, and statin use, HDL cholesterol tended to be negatively associated with change in BMLs.

Similar results were seen when area change at all four sites (medial tibial, medial femoral, lateral tibial, and lateral femoral) was used instead of total area change. This demonstrates consistency in our findings, showing that energy, carbohydrate, and sugar intake were positively associated with a BML change; and HDL cholesterol tended to be negatively associated with BML change.

No differences were found in the relation between dietary factors and lipids with change in BMLs in those with and without ROA, as no interaction terms with ROA were significant.

\section{Resolving BMLs}

Twenty-one subjects had complete BML resolution, and 147 subjects did not have complete BML resolution in those with a baseline BML. In a univariate analysis, HDL cholesterol was associated with resolving BMLs (OR, 1.67; $P=0.041$ ), and after adjustment for age, sex, BMI, ROA, and statin use, this remained significant (OR, 2.00; $P=0.027$ ). No dietary factors were associated with BML resolution.

\section{Incident BMLs}

The relation between baseline dietary factors and lipids and incident BMLs is presented in Table 4. Fourteen incident BMLs occurred across all four sites (medial tibial, medial femoral, lateral tibial, and lateral femoral). Total fat intake was protective against incident BMLs after adjustment for age, sex, BMI, and ROA. Saturated fat was also protective against incident BMLs in a multivariable model. In regard to serum lipids, HDL cholesterol was protective against incident BMLs after adjustment for age, sex, BMI, ROA, and statin use.

In a separate analysis that did not define incident BMLs by $25 \mathrm{~mm}^{2}$ (that is, including all those who developed a BML of any size; $n=24$ ), our results are largely 
Table 2 The association between baseline BMLs, dietary factors, and lipids ${ }^{\mathrm{a}}$

\begin{tabular}{|c|c|c|c|c|}
\hline & \multicolumn{2}{|l|}{ Univariate } & \multicolumn{2}{|l|}{ Multivariable $^{\mathbf{b}}$} \\
\hline & OR $(95 \% \mathrm{Cl})$ & $P$ & OR $(95 \% \mathrm{Cl})$ & $P$ \\
\hline \multicolumn{5}{|l|}{ Dietary factors } \\
\hline Energy intake & $1.12(0.91,1.36)$ & 0.285 & $1.07(0.84,1.35)$ & 0.592 \\
\hline Total fat & $1.09(0.89,1.33)$ & 0.385 & $1.06(0.84,1.34)$ & 0.630 \\
\hline Carbohydrate & $1.14(0.93,1.39)$ & 0.206 & $1.08(0.85,1.36)$ & 0.525 \\
\hline Protein & $1.07(0.88,1.30)$ & 0.517 & $1.03(0.82,1.29)$ & 0.785 \\
\hline Sugars & $1.13(0.93,1.39)$ & 0.217 & $1.11(0.89,1.39)$ & 0.344 \\
\hline \multicolumn{5}{|l|}{ Individual fats } \\
\hline Monounsaturated fat & $1.07(0.88,1.31)$ & 0.502 & $1.02(0.81,1.29)$ & 0.849 \\
\hline Polyunsaturated fat & $0.96(0.78,1.17)$ & 0.672 & $0.92(0.73,1.15)$ & 0.455 \\
\hline Saturated fat & $1.14(0.94,1.40)$ & 0.187 & $1.14(0.90,1.44)$ & 0.283 \\
\hline \multicolumn{5}{|l|}{ Lipids } \\
\hline Total cholesterol & $0.76(0.61,0.95)$ & 0.015 & $0.81(0.63,1.02)$ & $0.074^{c}$ \\
\hline Triglycerides & $1.00(0.82,1.22)$ & 0.996 & $0.90(0.72,1.13)$ & $0.384^{c}$ \\
\hline LDL cholesterol & $0.84(0.68,1.03)$ & 0.101 & $0.88(0.69,1.11)$ & $0.280^{c}$ \\
\hline HDL cholesterol & $0.77(0.62,0.95)$ & 0.016 & $0.89(0.70,1.14)$ & $0.357^{c}$ \\
\hline
\end{tabular}

${ }^{\mathrm{a} B M L}$ absent (226) versus present (168). Odds ratios (ORs) have been standardized. ${ }^{\mathrm{b}}$ Adjusted for age, sex, body mass index, smoking, cardiovascular diseases, diabetes, and radiographic osteoarthritis. ${ }^{C}$ Further adjusted for statin use. Boldface denotes a statistically significant result. BMLs, bone marrow lesions; $95 \% \mathrm{Cl}$ $95 \%$ confidence interval; $\mathrm{HDL}$, high-density lipoprotein; $\mathrm{LDL}$, low-density lipoprotein; $P, P$ value.

consistent but appeared weaker, suggesting that measurement error increased when we did not define incident BMLs by $25 \mathrm{~mm}^{2}$.

\section{Additional analysis}

Additional data on cartilage defects and meniscal pathology were available in this study. The dietary factors found to be associated with BML changes (energy, carbohydrate, and sugar intake) were not associated with cartilage defects, meniscal extrusion, or meniscal tears cross-sectionally (all $P>0.05$ ) or longitudinally (all $P>0.05$ ) in univariate and multivariable analysis, adjusting for age, sex, BMI, baseline BMLs, smoking, cardiovascular diseases, diabetes, and ROA. HDL cholesterol was also not associated with cartilage defects, meniscal extrusion, or meniscal tears cross-sectionally (all $P>$ 0.05 ) or longitudinally (all $P>0.05$ ) in univariate and multivariable analysis adjusting for age, sex, BMI,

Table 3 The association between dietary factors and lipids with a change in total BML size

\begin{tabular}{|c|c|c|c|c|}
\hline & \multicolumn{2}{|l|}{ Univariate } & \multicolumn{2}{|l|}{ Multivariable $^{a}$} \\
\hline & $\beta(95 \% \mathrm{Cl})$ & $P$ & $\beta(95 \% \mathrm{Cl})$ & $P$ \\
\hline \multicolumn{5}{|l|}{ Dietary factors } \\
\hline Energy intake & $8.60(-2.46,19.67)$ & 0.127 & $15.44(1.71,29.16)$ & 0.028 \\
\hline Total fat & $1.86(-9.24,12.95)$ & 0.742 & $7.70(-6.69,22.09)$ & 0.293 \\
\hline Carbohydrate & $14.45(3.45,25.46)$ & 0.010 & $19.27(6.23,32.31)$ & 0.004 \\
\hline Protein & $5.32(-5.76,16.41)$ & 0.346 & $12.00(-1.80,25.79)$ & 0.088 \\
\hline Sugars & $14.53(3.52,25.53)$ & 0.010 & $16.90(4.38,29.42)$ & 0.008 \\
\hline \multicolumn{5}{|l|}{ Individual fats } \\
\hline Monounsaturated fat & $3.37(-7.73,14.46)$ & 0.551 & $10.00(-4.50,24.50)$ & 0.176 \\
\hline Polyunsaturated fat & $6.26(-4.83,17.34)$ & 0.268 & $11.17(-1.85,24.20)$ & 0.092 \\
\hline Saturated fat & $-1.88(-12.97,9.22)$ & 0.740 & $1.37(-12.49,15.22)$ & 0.846 \\
\hline \multicolumn{5}{|l|}{ Lipids } \\
\hline Total cholesterol & $-5.45(-16.62,5.72)$ & 0.338 & $-6.02(-18.85,6.82)$ & $0.357^{b}$ \\
\hline Triglycerides & $-7.24(-18.42,3.95)$ & 0.204 & $-6.40(-19.05,6.25)$ & $0.321^{b}$ \\
\hline LDL cholesterol & $1.62(-9.59,12.83)$ & 0.776 & $-0.97(-14.27,12.32)$ & $0.885^{b}$ \\
\hline HDL cholesterol & $-11.18(-22.30,-0.05)$ & 0.049 & $-11.66(-25.08,1.76)$ & $0.088^{b}$ \\
\hline
\end{tabular}

Values are expressed as a change in total BML size $\left(\mathrm{mm}^{2}\right)$ per 1 standard deviation increase in dietary or lipid factor. ${ }^{a}$ Adjusted for age, sex, body mass index, baseline BMLs, smoking, cardiovascular disease, diabetes, radiographic osteoarthritis, and time to follow-up. ${ }^{b}$ Further adjusted for statin use. Boldface denotes statistically significant result. $95 \% \mathrm{Cl}, 95 \%$ confidence interval; $\beta$, beta coefficients; BML, bone marrow lesion; HDL, high-density lipoprotein; LDL, low-density lipoprotein; $P, P$ value. 
Table 4 The association between baseline dietary factors and lipids with incident BMLs ${ }^{\mathrm{a}}$

\begin{tabular}{|c|c|c|c|c|}
\hline & \multicolumn{2}{|l|}{ Univariate } & \multicolumn{2}{|l|}{ Multivariable $^{\mathbf{b}}$} \\
\hline & OR $(95 \% \mathrm{Cl})$ & $P$ & OR $(95 \% \mathrm{Cl})$ & $P$ \\
\hline \multicolumn{5}{|l|}{ Dietary factors } \\
\hline Energy intake & $0.68(0.34,1.37)$ & 0.282 & $0.64(0.29,1.39)$ & 0.257 \\
\hline Total fat & $0.40(0.17,0.93)$ & 0.034 & $0.32(0.12,0.86)$ & 0.023 \\
\hline Carbohydrate & $0.96(0.53,1.71)$ & 0.877 & $0.95(0.51,1.75)$ & 0.857 \\
\hline Protein & $0.79(0.38,1.67)$ & 0.542 & $0.78(0.35,1.75)$ & 0.552 \\
\hline Sugars & $0.98(0.54,1.75)$ & 0.933 & $0.96(0.52,1.73)$ & 0.856 \\
\hline \multicolumn{5}{|l|}{ Individual fats } \\
\hline Monounsaturated fat & $0.49(0.22,1.09)$ & 0.080 & $0.41(0.16,1.04)$ & 0.061 \\
\hline Polyunsaturated fat & $0.82(0.44,1.52)$ & 0.523 & $0.82(0.42,1.57)$ & 0.540 \\
\hline Saturated fat & $0.29(0.11,0.78)$ & 0.014 & $0.24(0.08,0.72)$ & 0.010 \\
\hline \multicolumn{5}{|l|}{ Lipids } \\
\hline Total cholesterol & $0.81(0.44,1.46)$ & 0.479 & $0.67(0.33,1.35)$ & $0.262^{c}$ \\
\hline Triglycerides & $0.69(0.33,1.45)$ & 0.329 & $0.75(0.34,1.65)$ & $0.474^{c}$ \\
\hline LDL cholesterol & $1.18(0.67,2.07)$ & 0.570 & $1.05(0.55,1.98)$ & $0.886^{c}$ \\
\hline HDL cholesterol & $0.52(0.27,1.03)$ & 0.060 & $0.34(0.14,0.78)$ & $0.011^{c}$ \\
\hline
\end{tabular}

${ }^{a}$ No BML present at baseline and follow-up $(n=212)$ versus incident BML $(n=14)$ at any site (medial tibial, medial femoral, lateral tibial, and lateral femoral), Odds ratios (ORs) have been standardized. ${ }^{b}$ Adjusted for age, sex, body mass index, and radiographic osteoarthritis. ${ }^{c}$ Further adjusted for statin use. Boldface denotes a statistically significant result. BML, bone marrow lesion; $95 \% \mathrm{Cl}, 95 \%$ confidence interval; $\mathrm{HDL}$, high-density lipoprotein; $\mathrm{LDL}$, low-density lipoprotein; $P$, $P$ value.

baseline BMLs, smoking, cardiovascular diseases, diabetes, ROA, and statin use.

Information also was available about physical activity. When the BML models were further adjusted for baseline steps/day, the results were largely unchanged.

\section{Discussion}

This longitudinal study reports associations between dietary factors, serum lipids, and BMLs. Despite an absence of cross-sectional associations, baseline energy, carbohydrate, and sugar intake were associated with increases in BML size. HDL cholesterol tended to be associated with a decrease in BML size and was protective against incident BMLs and associated with BML resolution. Total fat and saturated fat intake was protective against incident BMLs.

BMLs are associated with malalignment [3,9], increased loading [39], and increased body weight [40,41], emphasizing the relation between mechanical loading and BMLs. However, recent studies have suggested associations between BMLs and dietary fat intake $[16,17]$, suggesting a systemic role in BML pathology. Although we did not find any associations between fat intake and BML change, an exploratory analysis examining other dietary factors showed interesting findings. Total energy, carbohydrate, and sugar intake were positively associated with BML change. This suggests that an increase in these dietary factors may be detrimental to BMLs. Carbohydrate and sugar intake could be surrogate measures for increasing energy, and it seems most likely that increased energy is the major contributor to
BMLs. Evidence shows that a high-energy diet increases free radical production and oxidative stress $[42,43]$, which could be having an effect on BMLs. Additionally, the association we found was on a continuous scale; therefore, decreased energy, carbohydrate, and sugar intake was associated with BML decreases. Relating to this, evidence also shows that caloric restriction has antioxidative and antiinflammatory vasoprotective effects in both animal and human studies $[44,45]$. Therefore, a role may exist for dietary modification to alter BML natural history. However, the relation we report between dietary factors and BMLs may not be causal, and it may be that factors associated with diet are the underlying cause (for example, physical activity). In this study, we also collected information about physical-activity levels in the form of steps/day measured by pedometers for 2 weeks per year. This is a good reflection of usual physical activity $[46,47]$. When we further adjusted for steps/ day in the analyses, the results were largely unchanged, suggesting that the association is independent of physical activity. However, it is possible that other unknown confounders exist. Overall, these findings are novel and hypothesis generating, but must be confirmed in other studies.

To compare with previous studies, we did a separate analysis examining only incident BMLs. Total fat and saturated fat intake was protective against incident BMLs. These findings were unexpected and inconsistent. We found no associations between fat and BMLs in the cross-sectional analysis (Table 2) or in the longitudinal analysis (Table 3), in which we used a continuous 
measure of BML change. Traditionally, longitudinal studies are superior for demonstrating causal relations; however, our results also conflict with a recent longitudinal study by Wang et al. [17], which showed that increased saturated fat is associated with incident BMLs. These differences could reflect multiple comparisons in our current study. Alternatively, the discrepancy may reflect differences among study samples. In the study by Wang et al. [17] participants included only those without clinical signs of knee OA. Furthermore, the collection of dietary-intake data in the study by Wang et al. [17] occurred 10 to 14 years before the study period, rather than immediately before. It is possible that significant alterations in dietary behavior occurred within this time frame; however, some evidence indicates that nutrient intake is relatively stable and tends to be more stable with increasing age $[48,49]$. Most important, we only had 14 incident BMLs. However, with relatively few incident cases, we still see significant findings. Incident BMLs are a rare outcome, and the previous study by Wang et al. [17] also had a low number of incident BMLs $(n=32)$. It does seem logical that fat intake could be detrimental to BML development, given the histology of BMLs and the recent evidence linking them to vascular disease [29]. Therefore, our finding that fat is protective is surprising, and further work should be done to explore fat intake with BML development and progression. Specifically, now given the discrepant findings reported for fat intake and incident BMLs, we suggest that this should be explored in different study samples, with and without clinical knee OA. Interventional trials are also an option, as they will better control for unmeasured confounders. This could be done in both animal and human studies. To conclude, the current available evidence indicates that fat plays a role in BML development; however, the direction of the effect is unclear, and larger studies must verify our finding that fat is protective against incident BMLs.

This study has shown inconsistencies with dietary factors other than fat. Total energy and carbohydrate and sugar intake were associated with BML change but showed no association with incident BMLs. The analysis with dietary components including energy, carbohydrate, protein, and sugar intake was hypothesis generating and suggests that the association between diet and BMLs is different for incident BMLs versus progressing BMLs. However, this must be verified in future studies.

A recent study by Davies-Tuck et al. [29] found that serum cholesterol and triglycerides were associated with an increased incidence of BMLs in asymptomatic women. In the current study, we did not find any associations between total cholesterol, triglycerides, and BMLs; however, we did find that HDL cholesterol tended to be associated with a decrease in BML size.
In separate analyses, we found that HDL cholesterol was protective against incident BML development and associated with BML resolution. This provides consistent evidence that HDL cholesterol may have positive effects on BML pathology. It has been previously postulated that atheromatous vascular disease may contribute to the development of OA through its effects on subchondral bone [18]. Subchondral bone ischemia may be one mechanism by which vascular pathology contributes to the development of BMLs [19]. HDL cholesterol is considered to be protective against vascular pathology through cholesterol transport, antiinflammatory, and antioxidant effects [50], and therefore may help to reduce BML development and progression.

In this study, we were able to examine the effect of both increasing and decreasing BMLs, which other studies in the past have not been able to do $[17,29]$. The results were independent of potential confounders, such as smoking, cardiovascular diseases, diabetes, ROA, steps/day, and statin use. However, some limitations to this study exist. First, a 2-D assessment of BMLs was made, by using the slice with the greatest BML size. This may bias toward shallow but flat lesions. The majority of previous studies also grade BMLs on the slice with the greatest BML size; however, they use a semiquantitative scale (0 to 3 ) rather than an areal measure. We acknowledge that our measure of BMLs is only a surrogate measure of volume. Recent methods have been developed to measure BML volume by using a autoregression model [51,52]. It is our view that the slice thickness $(4 \mathrm{~mm})$ and interslice gap (0.5 to 1.0 $\mathrm{mm}$ ) of our imaging protocol were too large to estimate volume with sufficient accuracy. Although our areal measure may contain some measurement error, it has been demonstrated to be more sensitive to change over time, compared with a semiquantitative measure of BMLs [53]. Second, we did not measure knee alignment, which has been shown to be associated with BMLs $[3,9]$. Third, because of a small number of incident BMLs ( $n$ $=14$ ), we consider our finding that fat is protective against incident BMLs preliminary and must be reproduced in a larger study. Last, baseline and follow-up MRIs were read paired with the chronologic order known to the observer. This was done to identify the same lesion at follow-up. With few exceptions, the reader was confident that the same BML was assessed at follow-up. If a different BML were assessed at follow-up (that is, because it was the largest at that site at followup), this would increase our measurement error and dilute the effects we are seeing. However, as stated earlier, this was very rare, and in most cases, the reader was able to assess the same BML at baseline and followup. 


\section{Conclusion}

In conclusion, energy, carbohydrate, and sugar intake may be risk factors for BML development and progression. Fat was protective against incident BMLs, which is inconsistent with previous work and requires verification in other studies. HDL cholesterol appears to have protective effects on BMLs. Overall, this study suggests that macronutrients and serum lipids may be important in BML etiology. Multiple mechanisms exist by which diet and lipids could contribute to BML pathology, and further investigation into the relation between diet, lipids, and BMLs is warranted.

\section{Abbreviations}

2-D: two-dimensional; $\beta$ : beta coefficient; BMl: body mass index; BMLs: bone marrow lesions; Cl: confidence interval; FFQ: food-frequency questionnaire; HDL: high-density lipoprotein; LDL: low-density lipoprotein; LSC: least significant criterion; MRI: magnetic resonance imaging; OA: osteoarthritis; OR: odds ratio; ROA: radiographic osteoarthritis; SD: standard deviation; TASOAC: Tasmanian Older Adult Cohort Study.

\section{Acknowledgements}

We thank the subjects who made this study possible, and Catrina Boon and Pip Boon for their role in collecting the data. We thank Drs $V$ Parameswaran and J Burgess for laboratory measures. This work was supported by the National Health and Medical Research Council of Australia; Tasmanian Community Fund; Masonic Centenary Medical Research Foundation; Royal Hobart Hospital Research Foundation; and Arthritis Foundation of Australia.

\section{Author details}

${ }^{1}$ Menzies Research Institute Tasmania, University of Tasmania, Private Bag 23, Hobart, Tasmania, 7000, Australia. ${ }^{2}$ Cancer Epidemiology Centre, The Cancer Council of Victoria, Rathdowne Street, Carlton, Victoria, 3053, Australia. ${ }^{3}$ Department of Epidemiology and Preventive Medicine, Monash University, 89 Commercial Road, Melbourne, Victoria, 3004, Australia.

\section{Authors' contributions}

$\mathrm{DD}$ and $\mathrm{JdH}$ are co-first authors of this article. They were responsible for data management and cleaning, carried out analysis and interpretation of data, prepared the initial manuscript draft, and completed manuscript revisions. DD also collected data for this article. GG contributed to the conception and design of the study and critically revised the manuscript. CD designed and carried out the study planning, participated in analysis and interpretation of data, and critically revised the manuscript. FC designed and carried out the study planning, participated in analysis and interpretation of data, and critically revised the manuscript. GJ designed and carried out the study planning, participated in analysis and interpretation of the data, assisted with the initial manuscript draft, and critically revised the manuscript. All authors read and approved the final manuscript.

\section{Competing interests}

The authors declare that they have no competing interests.

Received: 29 July 2011 Revised: 20 December 2011

Accepted: 18 January 2012 Published: 18 January 2012

\section{References}

1. Lajeunesse D, Reboul P: The role of bone in the development of osteoarthritis. In Bone and Osteoarthritis. Edited by: Bronner F, FarachCarson M. London: Springer; 2007:19-39.

2. Felson DT, Chaisson CE, Hill CL, Totterman SM, Gale ME, Skinner KM, Kazis L, Gale DR: The association of bone marrow lesions with pain in knee osteoarthritis. Ann Intern Med 2001, 134:541-9.

3. Felson DT, McLaughlin S, Goggins J, LaValley MP, Gale ME, Totterman S, Li W, Hill C, Gale D: Bone marrow edema and its relation to progression of knee osteoarthritis. Ann Intern Med 2003, 139:330-6.
4. Zhai G, Blizzard L, Srikanth V, Ding C, Cooley H, Cicuttini F, Jones G: Correlates of knee pain in older adults: Tasmanian Older Adult Cohort Study. Arthritis Rheum 2006, 55:264-71.

5. Felson DT, Niu J, Guermazi A, Roemer F, Aliabadi P, Clancy M, Torner J, Lewis CE, Nevitt MC: Correlation of the development of knee pain with enlarging bone marrow lesions on magnetic resonance imaging. Arthritis Rheum 2007, 56:2986-92.

6. Dore D, Quinn S, Ding C, Winzenberg T, Zhai G, Cicuttini F, Jones G: Natural history and clinical significance of MRI-detected bone marrow lesions at the knee: a prospective study in community dwelling older adults. Arthritis Res Ther 2010, 12:R223.

7. Wluka AE, Wang Y, Davies-Tuck M, English DR, Giles GG, Cicuttini FM: Bone marrow lesions predict progression of cartilage defects and loss of cartilage volume in healthy middle-aged adults without knee pain over 2 years. Rheumatology (Oxford) 2008, 47:1392-6.

8. Dore D, Martens A, Quinn S, Ding C, Winzenberg T, Zhai G, Pelletier JP, Martel-Pelletier J, Abram F, Cicuttini F, Jones G: Bone marrow lesions predict site-specific cartilage defect development and volume loss: a prospective study in older adults. Arthritis Res Ther 2010, 12:R222.

9. Hunter DJ, Zhang Y, Niu J, Goggins J, Amin S, LaValley MP, Guermazi A, Genant H, Gale D, Felson DT: Increase in bone marrow lesions associated with cartilage loss: a longitudinal magnetic resonance imaging study of knee osteoarthritis. Arthritis Rheum 2006, 54:1529-35.

10. Pelletier JP, Raynauld JP, Berthiaume MJ, Abram F, Choquette D, Haraoui B, Beary JF, Cline GA, Meyer JM, Martel-Pelletier J: Risk factors associated with the loss of cartilage volume on weight-bearing areas in knee osteoarthritis patients assessed by quantitative magnetic resonance imaging: a longitudinal study. Arthritis Res Ther 2007, 9:R74.

11. Tanamas SK, Wluka AE, Pelletier JP, Pelletier JM, Abram F, Berry PA, Wang Y, Jones G, Cicuttini FM: Bone marrow lesions in people with knee osteoarthritis predict progression of disease and joint replacement: a longitudinal study. Rheumatology (Oxford) 2010, 49:2413-9.

12. Goggs R, Vaughan-Thomas A, Clegg PD, Carter SD, Innes JF, Mobasheri A, Shakibaei M, Schwab W, Bondy CA: Nutraceutical therapies for degenerative joint diseases: a critical review. Crit Rev Food Sci Nutr 2005 45:145-64.

13. Wang $Y$, Prentice LF, Vitetta $L$, Wluka AE, Cicuttini FM: The effect of nutritional supplements on osteoarthritis. Altern Med Rev 2004, 9:275-96.

14. Plumb MS, Aspden RM: High levels of fat and ( $n-6)$ fatty acids in cancellous bone in osteoarthritis. Lipids Health Dis 2004, 3:12.

15. Curtis CL, Rees SG, Little CB, Flannery CR, Hughes CE, Wilson C, Dent CM, Otterness IG, Harwood JL, Caterson B: Pathologic indicators of degradation and inflammation in human osteoarthritic cartilage are abrogated by exposure to n-3 fatty acids. Arthritis Rheum 2002, 46:1544-53.

16. Wang Y, Wluka AE, Hodge AM, English DR, Giles GG, O'Sullivan R, Cicuttini FM: Effect of fatty acids on bone marrow lesions and knee cartilage in healthy, middle-aged subjects without clinical knee osteoarthritis. Osteoarthritis Cartilage 2008, 16:579-83.

17. Wang Y, Davies-Tuck ML, Wluka AE, Forbes A, English DR, Giles GG, O'Sullivan R, Cicuttini FM: Dietary fatty acid intake affects the risk of developing bone marrow lesions in healthy middle-aged adults without clinical knee osteoarthritis: a prospective cohort study. Arthritis Res Ther 2009, 11:R63.

18. Conaghan $P G$, Vanharanta $H$, Dieppe PA: Is progressive osteoarthritis an atheromatous vascular disease? Ann Rheum Dis 2005, 64:1539-41.

19. Findlay DM: Vascular pathology and osteoarthritis. Rheumatology (Oxford) 2007, 46:1763-8.

20. Kadam UT, Jordan K, Croft PR: Clinical comorbidity in patients with osteoarthritis: a case-control study of general practice consulters in England and Wales. Ann Rheum Dis 2004, 63:408-14.

21. Singh G, Miller JD, Lee FH, Pettitt D, Russell MW: Prevalence of cardiovascular disease risk factors among US adults with self-reported osteoarthritis: data from the Third National Health and Nutrition Examination Survey. Am J Manag Care 2002, 8:S383-91.

22. Winet H, Hsieh A, Bao JY: Approaches to study of ischemia in bone. J Biomed Mater Res 1998, 43:410-21.

23. Zanetti M, Bruder E, Romero J, Hodler J: Bone marrow edema pattern in osteoarthritic knees: correlation between MR imaging and histologic findings. Radiology 2000, 215:835-40. 
24. Plenk H Jr, Hofmann S, Eschberger J, Gstettner M, Kramer J, Schneider W, Engel A: Histomorphology and bone morphometry of the bone marrow edema syndrome of the hip. Clin Orthop Relat Res 1997, 334:73-84.

25. Bergman AG, Willen HK, Lindstrand AL, Pettersson HT: Osteoarthritis of the knee: correlation of subchondral MR signal abnormalities with histopathologic and radiographic features. Skeletal Radiol 1994, 23:445-8.

26. Hunter DJ, Gerstenfeld L, Bishop G, Davis AD, Mason ZD, Einhorn TA, Maciewicz RA, Newham P, Foster M, Jackson S, Morgan EF: Bone marrow lesions from osteoarthritis knees are characterized by sclerotic bone that is less well mineralized. Arthritis Res Ther 2009, 11:R11.

27. Leydet-Quilici H, Le Corroller T, Bouvier C, Giorgi R, Argenson JN, Champsaur P, Pham T, de Paula AM, Lafforgue P: Advanced hip osteoarthritis: magnetic resonance imaging aspects and histopathology correlations. Osteoarthritis Cartilage 2010, 18:1429-35.

28. Aaron RK, Dyke JP, Ciombor DM, Ballon D, Lee J, Jung E, Tung GA: Perfusion abnormalities in subchondral bone associated with marrow edema, osteoarthritis, and avascular necrosis. Ann N Y Acad Sci 2007, 1117:124-37.

29. Davies-Tuck ML, Hanna F, Davis SR, Bell RJ, Davison SL, Wluka AE, Adams J, Cicuttini FM: Total cholesterol and triglycerides are associated with the development of new bone marrow lesions in asymptomatic middleaged women: a prospective cohort study. Arthritis Res Ther 2009, 11:R181.

30. Giles GG, Ireland PD: Dietary Questionnaire for Epidemiological Studies (Version 2). Melbourne: The Cancer Council of Victoria; 1996.

31. Hodge A, Patterson AJ, Brown WJ, Ireland P, Giles G, The Anti Cancer Council of Victoria FFQ: Relative validity of nutrient intakes compared with weighed food records in young to middle-aged women in a study of iron supplementation. Aust N Z J Public Health 2000, 24:576-83.

32. Radimer K, Bindewald B, Hughes J, Ervin B, Swanson C, Picciano MF: Dietary supplement use by US adults: data from the National Health and Nutrition Examination Survey, 1999-2000. Am J Epidemiol 2004, 160:339-49.

33. Friedewald WT, Levy RI, Fredrickson DS: Estimation of the concentration of low-density lipoprotein cholesterol in plasma, without use of the preparative ultracentrifuge. Clin Chem 1972, 18:499-502.

34. Altman RD, Hochberg M, Murphy WA Jr, Wolfe F, Lequesne M: Atlas of individual radiographic features in osteoarthritis. Osteoarthritis Cartilage 1995, 3(Suppl A):3-70.

35. Dore D, Quinn S, Ding C, Winzenberg T, Jones G: Correlates of subchondral BMD: a cross-sectional study. J Bone Miner Res 2009, 24:2007-15.

36. Ding C, Garnero P, Cicuttini F, Scott F, Cooley H, Jones G: Knee cartilage defects: association with early radiographic osteoarthritis, decreased cartilage volume, increased joint surface area and type II collagen breakdown. Osteoarthritis Cartilage 2005, 13:198-205.

37. Berthiaume MJ, Raynauld JP, Martel-Pelletier J, Labonte F, Beaudoin G, Bloch DA, Choquette D, Haraoui B, Altman RD, Hochberg M, Meyer JM, Cline GA, Pelletier JP: Meniscal tear and extrusion are strongly associated with progression of symptomatic knee osteoarthritis as assessed by quantitative magnetic resonance imaging. Ann Rheum Dis 2005, 64:556-63.

38. Nguyen TV, Eisman JA: Assessment of significant change in BMD: a new approach. J Bone Miner Res 2000, 15:369-72.

39. Bennell KL, Creaby MW, Wrigley TV, Bowles KA, Hinman RS, Cicuttini F, Hunter DJ: Bone marrow lesions are related to dynamic knee loading in medial knee osteoarthritis. Ann Rheum Dis 2010, 69:1151-4.

40. Guymer E, Baranyay F, Wluka AE, Hanna F, Bell RJ, Davis SR, Wang Y, Cicuttini FM: A study of the prevalence and associations of subchondral bone marrow lesions in the knees of healthy, middle-aged women. Osteoarthritis Cartilage 2007, 15:1437-42.

41. Davies-Tuck ML, Wluka AE, Wang Y, English DR, Giles GG, Cicuttini F: The natural history of bone marrow lesions in community-based adults with no clinical knee osteoarthritis. Ann Rheum Dis 2009, 68:904-8.

42. Hennig B, Toborek M, McClain CJ: High-energy diets, fatty acids and endothelial cell function: implications for atherosclerosis. J Am Coll Nutr 2001, 20:97-105.

43. Devaraj S, Wang-Polagruto J, Polagruto J, Keen $\mathrm{CL}$, Jialal I: High-fat, energydense, fast-food-style breakfast results in an increase in oxidative stress in metabolic syndrome. Metabolism 2008, 57:867-70.

44. Csiszar A, Labinskyy N, Jimenez R, Pinto JT, Ballabh P, Losonczy G, Pearson KJ, de Cabo R, Ungvari Z: Anti-oxidative and anti-inflammatory vasoprotective effects of caloric restriction in aging: role of circulating factors and SIRT1. Mech Ageing Dev 2009, 130:518-27.

45. Hofer T, Fontana L, Anton SD, Weiss EP, Villareal D, Malayappan B, Leeuwenburgh $C$ : Long-term effects of caloric restriction or exercise on DNA and RNA oxidation levels in white blood cells and urine in humans. Rejuv Res 2008, 11:793-9.

46. Tudor-Locke C, Bassett DR Jr: How many steps/day are enough? Preliminary pedometer indices for public health. Sports Med 2004, 34:1-8.

47. Wyatt HR, Peters JC, Reed GW, Barry M, Hill JO: A Colorado statewide survey of walking and its relation to excessive weight. Med Sci Sports Exerc 2005, 37:724-30.

48. Fernyhough LK, Horwath CC, Campbell AJ, Robertson MC, Busby WJ: Changes in dietary intake during a 6-year follow-up of an older population. Eur J Clin Nutr 1999, 53:216-25.

49. Goldbohm RA, van 't Veer $P$, van den Brandt PA, van 't Hof MA, Brants HA, Sturmans F, Hermus RJ: Reproducibility of a food frequency questionnaire and stability of dietary habits determined from five annually repeated measurements. Eur J Clin Nutr 1995, 49:420-9.

50. Barter PJ, Nicholls S, Rye KA, Anantharamaiah GM, Navab M, Fogelman AM: Antiinflammatory properties of HDL. Circ Res 2004, 95:764-72.

51. Li X, Ma BC, Bolbos Rl, Stahl R, Lozano J, Zuo J, Lin K, Link TM, Safran M, Majumdar S: Quantitative assessment of bone marrow edema-like lesion and overlying cartilage in knees with osteoarthritis and anterior cruciate ligament tear using MR imaging and spectroscopic imaging at 3 Tesla. J Magn Reson Imaging 2008, 28:453-61.

52. Zhao J, Li X, Bolbos Rl, Link TM, Majumdar S: Longitudinal assessment of bone marrow edema-like lesions and cartilage degeneration in osteoarthritis using 3 T MR T1rho quantification. Skeletal Radiol 2010, 39:523-31.

53. Laslett LL, Dore D, Quinn S, Winzenberg T, Boon P, Jones G: A twelvemonth randomized controlled trial of zoledronic acid for knee pain and subchondral bone marrow lesions. Proceedings of the 5th International Workshop on Osteoarthritis Imaging: 8-11 June 2011; Salzburg 2011, 20.

\section{doi:10.1186/ar3689}

Cite this article as: Doré et al:: A longitudinal study of the association between dietary factors, serum lipids, and bone marrow lesions of the knee. Arthritis Research \& Therapy 2012 14:R13.

\section{Submit your next manuscript to BioMed Central and take full advantage of:}

- Convenient online submission

- Thorough peer review

- No space constraints or color figure charges

- Immediate publication on acceptance

- Inclusion in PubMed, CAS, Scopus and Google Scholar

- Research which is freely available for redistribution

Submit your manuscript at www.biomedcentral.com/submit
C Biomed Central 